\title{
Magneto-optical Visualization of Vortices Penetration into $\mathrm{Ba}\left(\mathrm{Fe}_{1.8} \mathrm{Co}_{0.2}\right) \mathrm{As}_{2}$
}

\author{
Zhi Wei Lin, Jian Guo Zhu, Youguang Guo, Yong Jian Li \\ Faculty of Engineering and Information Technology, University of Technology, Sydney, PO \\ Box 123, NSW 2007, Australia \\ Dr. Shuhong Wang \\ Faculty of Electrical Engineering, Xi'an Jiaotong University, Xi'an, 710049, China
}

\begin{abstract}
Vortices penetration into oxygen-free superconducting compounds FeAs-122 system is of interest in understanding superconductivity. This work studies the vortices motion in $\mathrm{Ba}\left(\mathrm{Fe}_{1.8} \mathrm{Co}_{0.2}\right) \mathrm{As}_{2}$ single crystal by means of magneto-optical imaging technique in zero field cooled and field cooled conditions. The captured magneto-optical images and corresponding flux profiles show that at zero field cooled condition vortices penetrate into the crystal from the edges as external magnetic field increases. A vortices-free region is observed at the center of sample as applied field is less than full penetration field. In field cooled condition, the pinned vortices leave the sample from the edges as field decreases and polarization of the vortices at the edges are opposite as decreasing field approaches to zero. The pinning strength is decreases with increasing temperature. The observed vortex behaviour is very similar to that in high $\mathrm{T}_{\mathrm{c}}$ superconducting materials with strong pinning strength.
\end{abstract}

\section{Introduction}

The recent synthesized rare earth layered oxypnictides, $\mathrm{REFeAsO}_{1-\mathrm{x}} \mathrm{F}_{\mathrm{x}}$ (FeAs-1111 system, RE: La, Pr, Ce, Sm) with transition temperature $\mathrm{T}_{c}$ up to $54 \mathrm{~K}^{1}$ for $\mathrm{RE}=\mathrm{Sm}$ refresh interest in superconductivity. Meanwhile, their oxygen-free parent compounds FeAs-122 system, for example, $\mathrm{BaFe}_{2} \mathrm{As}_{2}$ composition ${ }^{2}$ was report to have $\mathrm{T}_{\mathrm{c}}=38$ $\mathrm{K}$ by replacing the alkaline earth element with alkali elements. ${ }^{3,4}$ Vortices penetration into both systems is of interest in understanding vortices dynamics and comparing with high- $T_{c}$ copper oxides. Unfortunately, it is very difficulty to grow relatively large single crystal for FeAs1111 system. However, FeAs-122 system can be grown in a large single crystal in flux at ambient pressure.

SQUID and VSM are normally used to study magnetization process, but they only exhibit collective information. However, magneto-optical imaging technique can visualize local and real-time vortices motion in the sample. This paper presents magneto-optical images to show how vortices penetrate into a $\mathrm{Ba}\left(\mathrm{Fe}_{1.8} \mathrm{Co}_{0.2}\right) \mathrm{As}_{2}$ single crystal.

\section{Experiment detail}

Single crystals of superconducting $\mathrm{Ba}\left(\mathrm{Fe}_{1.8} \mathrm{Co}_{0.2}\right) \mathrm{As}_{2}$ was grown out of Fe-As flux using standard hightemperature solution growth technique. ${ }^{5}$ Critical temperature Tc $=25 \mathrm{~K}$. The sample is trapezoid shape with height of $1.4 \mathrm{~mm}$ and thickness of $0.1 \mathrm{~mm}$. The magnetooptical imaging (MOI) system based on the Faraday rotate with temperature down to $4.2 \mathrm{~K}$ and field up to 800 Oe was used to visualize vortices motion in the sample. An indicator film with in-plane magnetization was directly placed on the sample which was glued to a cold head of the MOI system. The advantage of the MOI is that the local vortices distribution can be visualized in $3 \mu \mathrm{m}$ in real time.

\section{Results and discussion}

Figure 1 shows magneto-optical (MO) images as external field increased to $40 \mathrm{Oe}(\mathrm{a}), 200 \mathrm{Oe}(\mathrm{b}), 400 \mathrm{Oe}(\mathrm{c})$, $800 \mathrm{Oe}(\mathrm{d})$ and decreased to $400 \mathrm{Oe}(\mathrm{e}), 200 \mathrm{Oe}(\mathrm{f}), 0$ Oe (g) after the sample was zero-field cooled (ZFC) to $4.2 \mathrm{~K}$. Figure 2 shows the corresponding flux profile along the red line in figure 1(d). The scratch in the indicator film cause unexpected drop at left side. It can be seen that the sample is in Meissnar state at $40 \mathrm{Oe}$, that is, the field is completely shielded inside the sample. With increasing the field vortices start penetration into the sample from the edges. The vortex-free region exists in the center of the sample. The flux front moves towards the center of the sample as field is increasing. Such front penetration is clearly shown in the profile. However, full penetration is not observed since the field generated by electromagnet is limited to 800 Oe, less than $\mathrm{H}_{\mathrm{a}}$ at which full penetration occurs. As field decreases, only the vortices along the edge are expelled from the edge, and vortices far to the edge are still pinned. The observed flux motion in the sample is similar to that in high-Tc superconducting materials and is well agree with Bean model ${ }^{6}$ and theoretical calculation. ${ }^{7,8}$ However, the theoretical peaks at the edges is not clearly observed in this work since the edges of sample are not well defined and a gap exists between the indicator film and sample surface is relatively large.

Figure 3 shows MO images while the sample was field cooled to $4.2 \mathrm{~K}$ in application of external field of $800 \mathrm{Oe}$, then the field reduced to zero. The corresponding flux profiled is plot in figure 4 . The flux density in the middle of the sample does not change much, however, it reduces quick towards both edges. It means that the vortices introduced above the Tc are pinned in the sample as temperature decreases, but they leave the sample following the field reduction. The density changes significantly at the sample edges as the field reduced to zero. The vortices even change polarization. This phenomenon is quite similar 
with the high-Tc copper oxides. With increasing the temperature, the pinning strength becomes weak. The vortices pinned at $4.2 \mathrm{~K}$ are expelled from the sample, as shown in figure 3(c) and (d). The flux density becomes weaker and weaker. At $25 \mathrm{~K}$ the sample loses superconductivity, being consistent with $\mathrm{T}_{\mathrm{c}}$.

\section{Conclusion}

Magneto-optical imaging system is used to study vortices motion in $\mathrm{Ba}\left(\mathrm{Fe}_{1.8} \mathrm{Co}_{0.2}\right) \mathrm{As}_{2}$ single crystal grown using high-temperature solution growth technique. It is found that the vortex motion in oxygen-free parent compounds FeAs-122 system is very similar to that in the high- $\mathrm{T}_{\mathrm{c}}$ copper oxide superconductor with strong pinning strength. At temperature below $T_{c}$, vortices penetrate into the sample from sample edges as applied field increases, forming a flux front. As field decreases the pinned vortices are expelled from the sample, forming a flux density gradient. The polarization of the vortices at the sample is opposition as applied field approaches to zero. The pinning strength is decreases with increasing temperature.

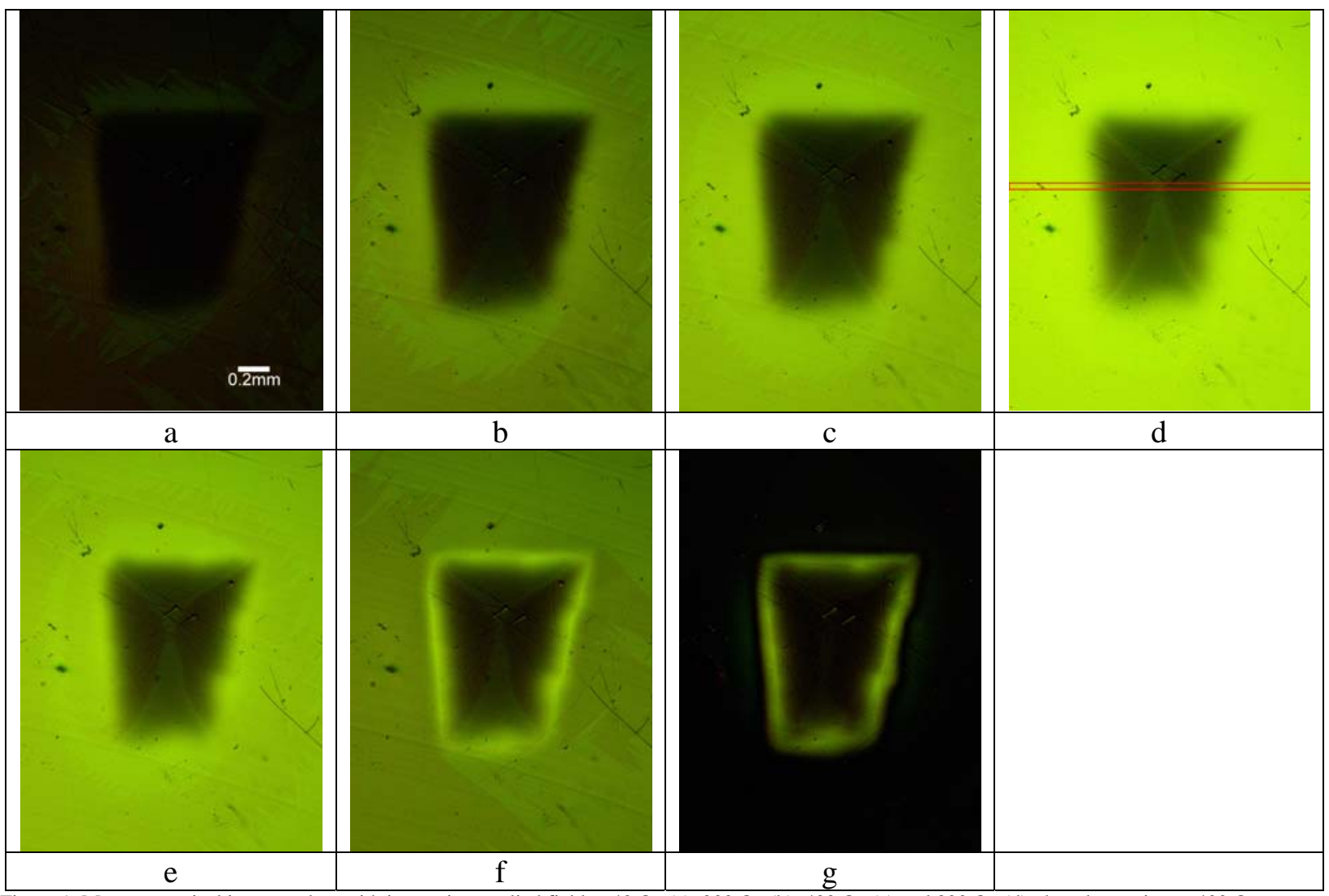

Figure 1. Magneto-optical images taken with increasing applied field at 40 Oe (a), 200 Oe (b), 400 Oe (c) and 800 Oe (d), then decreasing at 400 Oe (e), 200 Oe (f), and 0 Oe (g).
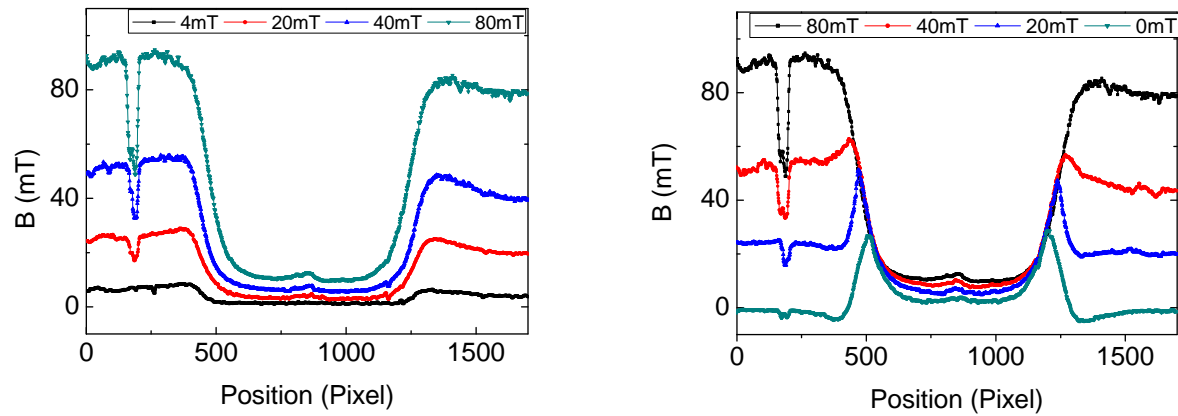

Figure 2. Flux profile corresponding to figure 1 with increasing field (a) and decreasing field (b). 


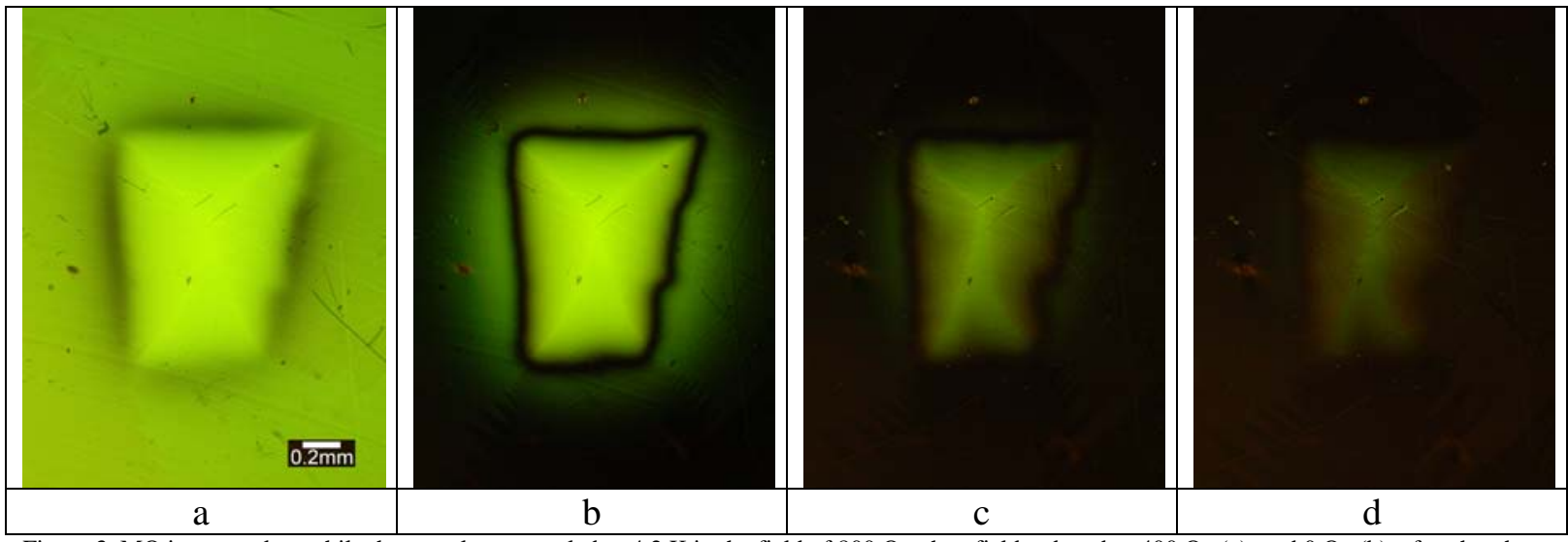

Figure 3. MO images taken while the sample was cooled to $4.2 \mathrm{~K}$ in the field of $800 \mathrm{Oe}$, then field reduced to 400 Oe (a), and 0 Oe (b). after that the temperature increased to $20 \mathrm{~K}$ (c) and $23 \mathrm{~K}(\mathrm{~d})$.

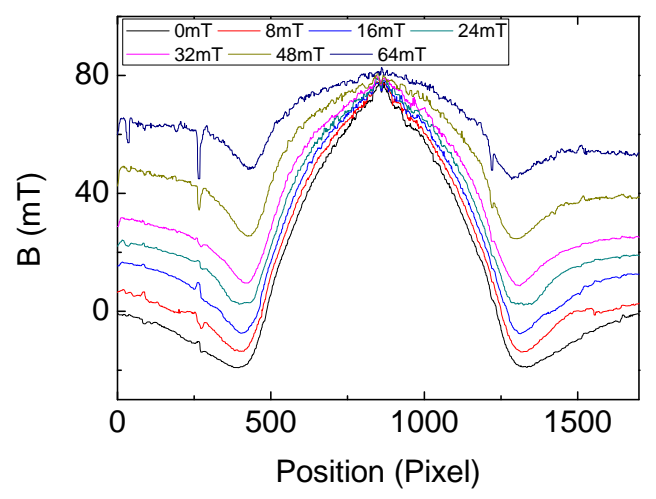

Figure 4. Flux profile for the sample FC to $4.2 \mathrm{~K}$ in $80 \mathrm{mT}$ following by reducing field to zero.

\section{Reference}

${ }^{1}$ Z. A. Ren, W. Lu, J. Yang, W. Yi, X. L. Shen, Z. C. Li, G. C. Che, X. L. Dong, L. L. Sun, F. Zhou, and Z. X. Zhao, Chinese Physics Letters, vol. 25, pp. 2215-2216, 2008.

${ }^{2}$ R. Marianne, T. Marcus, J. Dirk, S. Inga, H. Wilfried, and P. Rainer, Physical Review B, vol. 78, p. 020503, 2008.

${ }^{3}$ M. Rotter, M. Tegel, and D. Johrendt, Physical Review Letters, vol. 101, pp. 107006-4, 2008.

${ }^{4}$ K. Sasmal, B. Lv, B. Lorenz, A. M. Guloy, F. Chen, Y.-Y. Xue, and C.-W. Chu, Physical Review Letters, vol. 101, pp. 107007-4, 2008.

${ }^{5}$ Y. K. Li, X. Lin, T. Zhou, J. Q. Shen, Q. Tao, G. H. Cao, and Z. A. Xu, Journal of Physics-Condensed Matter, vol. 21, p. 5, Sep 2009.

${ }^{6}$ C. P. Bean, Reviews of Modern Physics, vol. 36, p. 31, 1964.

${ }^{7}$ T. Schuster, H. Kuhn, E. H. Brandt, M. Indenbom, M. R. Koblischka, and M. Konczykowski, Physical Review B-Condensed Matter, vol. 50, pp. 16684-707, 1994

${ }^{8}$ E. H. Brandt and M. Indenbom, Physical Review B, vol. 48, pp. 12893-906, 1993. 